\title{
Rare Entities of Mixed Phenotypic Acute Leukemia: Are They Really So Rare? Largest Series of B+T MPAL
}

\author{
*Raghvendra H Vijay1, "Namrata N Rajkumar', Vijay C Raghu², Chinnagiriyappa LK ${ }^{3}$ \\ ${ }^{1}$ Dr Raghavendra H .V. Associate Professor of Pathology, ${ }^{1}$ Dr Namrata N R ,Assistant Professor of Pathology, ${ }^{2}$ Vijay C. \\ Raghu, Assistant Professor, Department Biostatistics, ${ }^{3}$ Dr Lakshmiah K Chinnagiriyappa. Prof \& head Department of \\ Medical Oncology. All are affiliated with Kidwai Memorial Institute of Oncology Bangalore, Karnataka, India \\ *Dr Raghavendra H.V. and Namrata N R have contributed equally for study.
}

Address for Correspondence: Dr. Namrata N R, Assistant Professor, Pathology, KMIO, Bangalore, Karnataka, India. Email: nrnamrata@yahoo.com

\begin{abstract}
Background: MPAL are rare, accounts $4 \%$ of acute leukemias. B+T MPAL are extremely rare. There is no robust information on the clinical and biologic features of these leukemias. Present study from kidwai memorial institute of oncology focused on MPAL rare types, especially B+T MPAL. Design and Methods: We attempted to classify MPAL, based on WHO 2008 classification and attempted to summarise diagnostic criteria, cytochemistry, immunophenotyping, cytogenetics \& clinical features of B+T MPAL. Results: Most MPAL cases reported were B/Myeloid, followed by B+T MPAL, T+Myeloid, undifferentiated and unclassifiable leukemias respectively. Conclusion: Among MPAL unusual high incidence of B+T MPAL (38.4\%) was noted. Overall median survival was 5 years.
\end{abstract}

Keywords: Acute leukemias of ambiguous lineage, Mixed phenotypic acute leukemia, aberrant antigens, Lymphoid, cytogenetics, Flow immunophenotyping. MCP 841 Protocol.

\section{Introduction}

Acute leukemias of ambiguous lineage (ALAL) are rare leukemias recognized by WHO Classification of Tumors of Haematopoietic and Lymphoid tissue [2008]. The majority of these rare leukemias (ALAL) are classified as mixed phenotype acute leukemia (MPAL). When the blasts express more than one lineage antigens, assigning to particular lineage is not possible with certainty, it is termed MPAL. MPAL by definition doesn't fit into any category, either by clinical or genetic features [1,2]. MPAL accounts for $4 \%$ of Leukemias. Immunophenotypically Mixed phenotype acute leukemia can be classified as B/Myeloid, T/Myeloid, B+T MPAL or trilineage MPAL based on Immunophenotypic findings $[1,4]$. There is paucity of data regarding the precise biologic characterization of the disease as well as established guidelines on the management and prognosis of these patients' as these

Manuscript received: $22^{\text {nd }} \mathrm{Feb} 2016$

Reviewed: $27^{\text {th }}$ Feb 2016

Author Corrected: $10^{\text {th }}$ March 2016

Accepted for Publication: 22nd March 2016 leukemias are rare and the formal criteria for diagnosis have been established recently $[5,6$,$] . \mathrm{B}+\mathrm{T}$ MPAL are extremely uncommon, comprise only $4 \%$ of MPAL $[3,6]$. Present study of 10 cases of $\mathrm{B}+\mathrm{T}$ MPAL is the largest reported series of $\mathrm{B}+\mathrm{T}$ MPAL in literature till date.

\section{Material \& Methods}

Our laboratory received 1800 of acute leukemias, during a period of 4 years from January 2012 to February2016, where flow cytometric analysis was done for diagnosis. We followed WHO classification 2008 criterias along with, morphology, cytochemistry, immunophenotyping, cytogenetics and clinical findings. 31 patients were diagnosed as AL AL. Out of these 26 cases was MPAL. Study concentrated mainly on 10 cases of B+T MPAL. These leukemias were equally distributed in 5 children and 5 adults.

Treated Leukemias, that switch their lineage during therapy were not included in this study. All specimens 
were obtained and morphologic examination was done. Airdried peripheral smears and bone marrow aspirates were stained with Giemsa stain. Cytochemical Myeloperoxidase was done in all cases and Periodic acid-Schiff, nonspecific esterase and specific esterase accordingly if required.

Four color flow cytometry analysis was performed on Bone marrow aspirate using FACS caliber (Becton Dickinson. San Jose, CA, USA).CD45 vs side scatter gating strategy was used. Immunophenotype features are summarized in table 1

Cytogenetic analysis was performed on overnightunstimulated cultures of bone marrow, later followed by $\mathrm{G}$ banding.

Statistical methods: Probability of Event free survival was estimated using The Kaplan-Meier method. Petoetal. Method was used to determine SEs. survival comparison were performed with $\mathrm{R}$ software.

\section{Markers expressed inB+TMPAL (Table-1)}

\begin{tabular}{|c|c|c|c|}
\hline \multicolumn{4}{|c|}{$\frac{\text { Case B markers T cell markers Immature Markers }}{\text { Specific Other } \quad \text { Specific Other }}$} \\
\hline \multicolumn{4}{|r|}{ CD19 cCD79a, CD10 Cy CD3 CD5, CD7 } \\
\hline \multicolumn{4}{|r|}{ CD19 cCD79a Cy CD3 CD5, CD7 } \\
\hline \multicolumn{4}{|r|}{ CD19 cCD79a Cy CD3 CD5, CD7 TdT } \\
\hline \multicolumn{4}{|r|}{ CD19 cCD79a, CD10 Cy CD3 CD7 CD34 } \\
\hline \multicolumn{2}{|l|}{5} & & CD19 cCD79a Cy CD3 CD5, CD7 CD34 TdT \\
\hline \multicolumn{2}{|l|}{6} & & CD19 cCD79a, CD10, Cy CD3 CD5, CD34 TdT \\
\hline \multicolumn{2}{|l|}{7} & & CD19 cCD79a, CD10, Cy CD3 CD5, CD5, \\
\hline \multirow{2}{*}{\multicolumn{2}{|c|}{$\begin{array}{l}8 \\
9\end{array}$}} & & CD19 cCD79a, Cy CD3 CD5, CD7 CD34, \\
\hline & & & CD19 cCD79a, CD10, Cy CD3 CD5, CD7 CD34, TdT \\
\hline
\end{tabular}

\section{Results}

During the study period of 4-years, flowcytometric analyses of 1800 cases of acute leukemias were performed and a diagnosis of ALAL, was made in 31 patients (1.7\%). MPAL was diagnosed using WHO criteria in 26 patients $(1.4 \%) .5$ cases were acute undifferentiated leukemias/unclassifiable leukemias. Among 26 MPAL cases, 12 cases were B+MYELOID MPAL( 46\%), 4 cases were T+Myeloid MPAL.10cases of T+B MPAL (38.4\%), 5 were children $<15$ yrs, 5 were reported in adults.

Male preponderance noted in ALAL cases and MPAL cases. B+Myeloid MPAL and also B+TMPAL revealed male predominance. Ultrasonograghy in $2 / 10$ cases $(20 \%)$ revealed hepatosplenomegaly. Lymphadenopathy was noted in 4 / $10(40 \%)$ cases on clinical examination. Single case had mediastinal enlargement.Baseline investigations revealed leucocytosis in 5patients $(50 \%)$, low hemoglobin $(<10 \mathrm{~g} / \mathrm{dl})$ in 8 cases $(80 \%)$, and low platelet count in all patients.

Mean serum lactate dehydrogenase (LDH) was 1099IU/L, mean serum alkaline phosphatase was 215 IU/L and elevated liver enzymes in (30\%) cases. Serological studies for HIV, HBsAg were negative in all cases, except a single case was positive for HBsAg. Blasts percentage varied from $40 \%$ to $90 \%$.

All Cases of B+T MPAL showed lymphoid morphology, (FIG1) Table1 summarizes Immuno phenotypic findings. Flow cytometry scattergrams in fig2. Myeloperioxidase was negative both on flowcytometry and cytochemistry in all cases.

Marker of early hematopoietic cells, CD34, was strongly positive in5/10 cases (50\%). Tdt was positive for in 4 cases. cytogenetic data were available in all cases, 4 cases had normal karyotype, 4 cases did not yield any metaphase, 2 case showed abnormal karyotype and interpreted as hyperdiploid in one case,other case as malignant clone. 


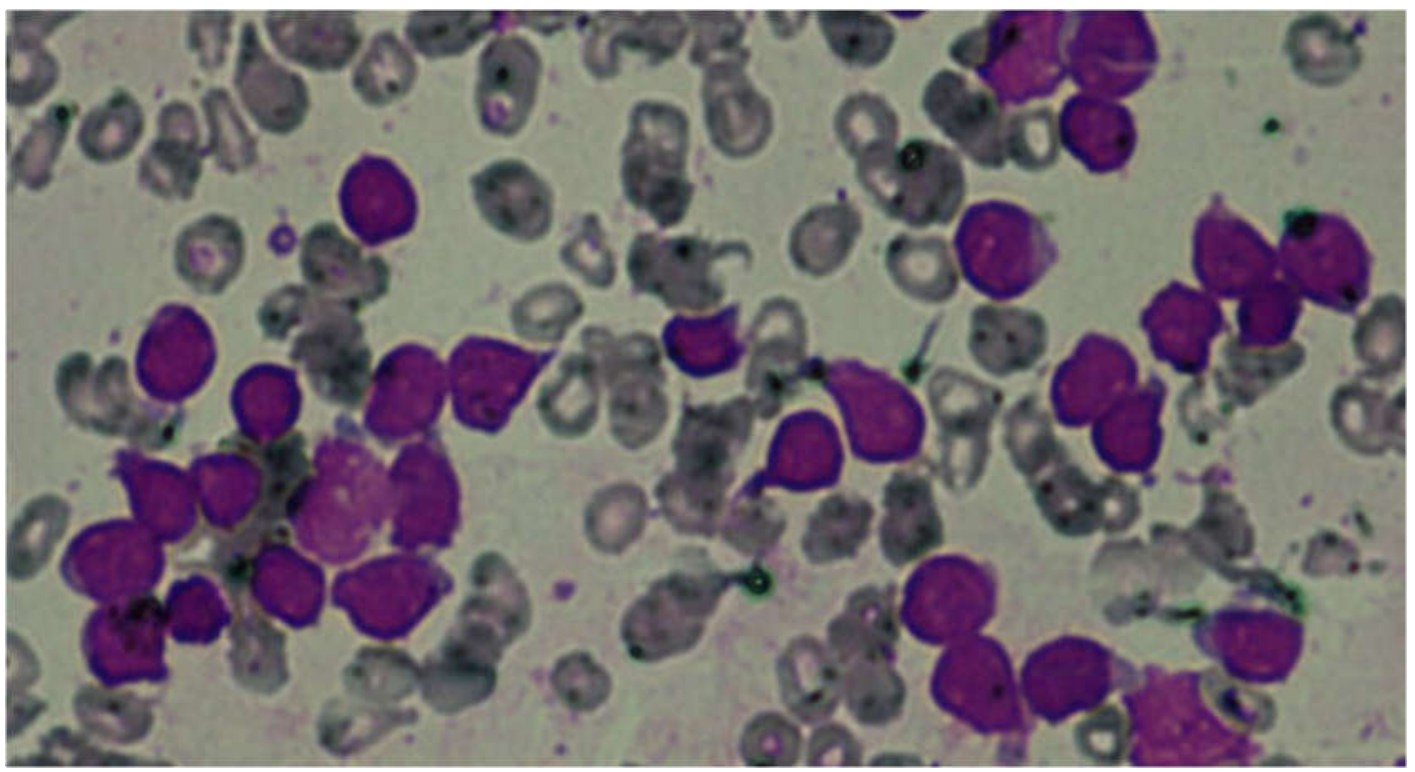

Fig 1: Lymphoblasts in B+T MPAL
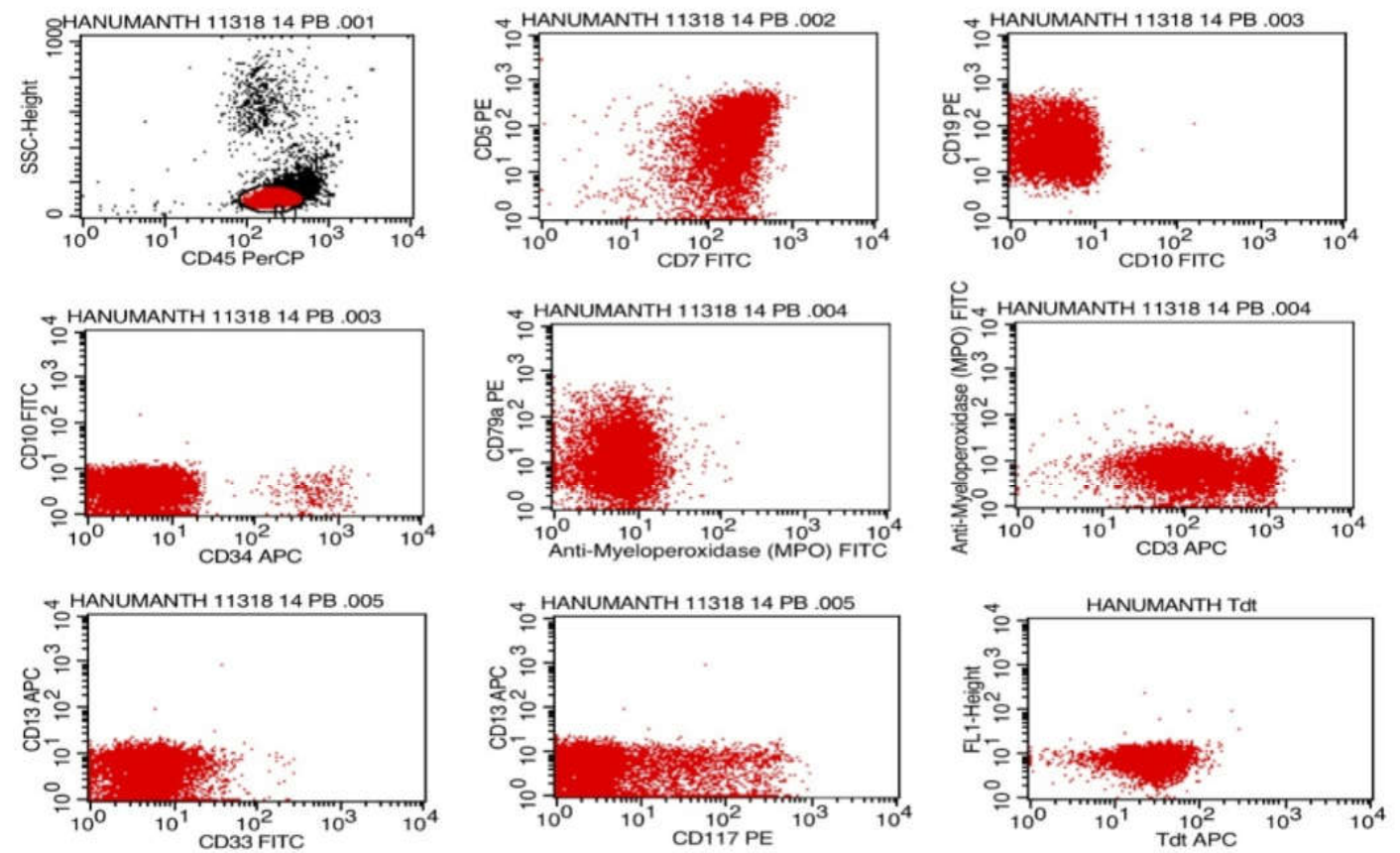

Gated cells in the blast region are

\# positive for Tdt, cCD3, CD5, CD7, CD19, cCD79a.

\# Negative for CD34, CD13, MPO, CD33, CD117, MPO.

Fig 2: $\mathrm{B}+\mathrm{T}$ MPAL

At the end of induction period morphological complete remission (CR) $(<5 \%$ blasts in the bone marrow aspiration smear) was achieved in all treated cases and 8 cases continue to be in remission for median 5 years. Patients were treated with ALL regimens based protocol, MCP841 protocol in children and BFM95 protocol in adults, single patient relapsed with testicular infilteration, followed by cutaneous infilteration and bone marrow involvement. Overall mean survival was 5 years (fig $3 \& 4$ ). 


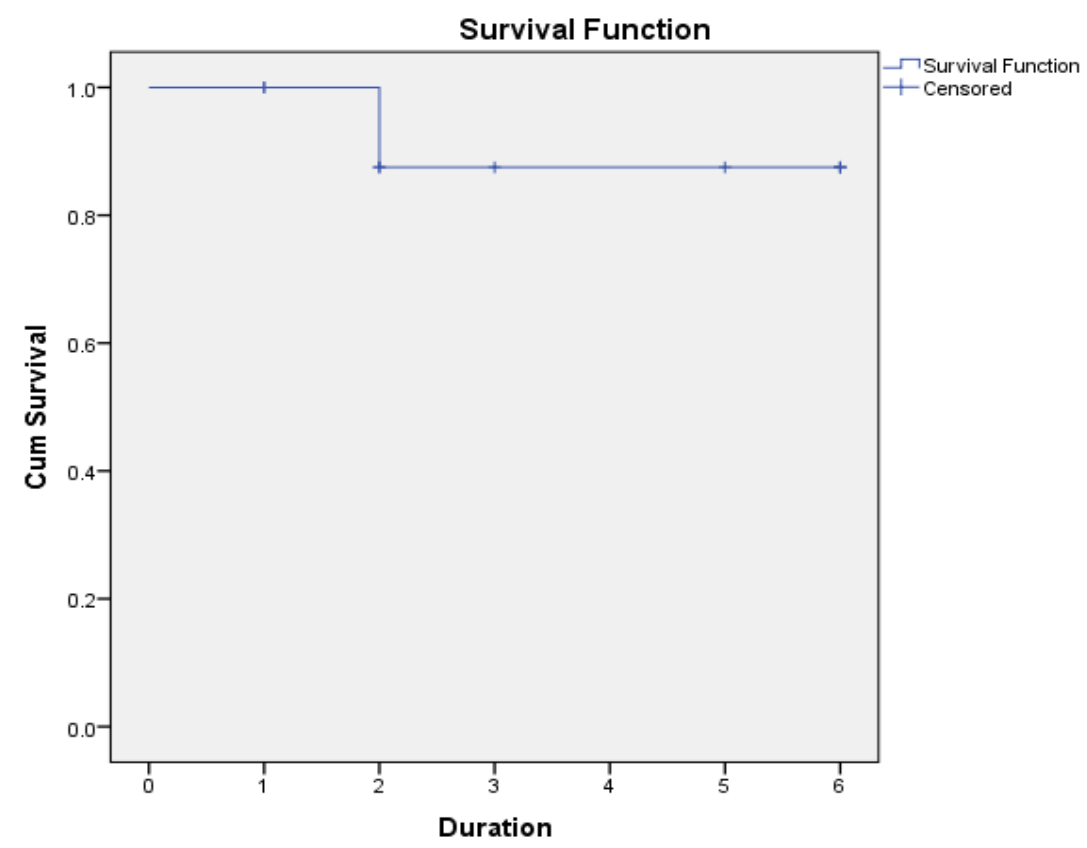

Fig: 3: Overall mean survival

\begin{tabular}{|c|c|c|c|c|c|c|c|}
\hline \multicolumn{8}{|c|}{ Means and Medians for Survival Time } \\
\hline \multicolumn{4}{|c|}{ Mean $^{\mathrm{a}}$} & \multicolumn{4}{|l|}{ Median } \\
\hline \multirow[b]{2}{*}{ Estimate } & \multirow[b]{2}{*}{ Std. Error } & \multicolumn{2}{|c|}{$95 \%$ Confidence Interval } & \multirow[b]{2}{*}{ Estimate } & \multirow[b]{2}{*}{ Std. Error } & \multicolumn{2}{|c|}{$95 \%$ Confidence Interva } \\
\hline & & $\begin{array}{l}\text { Lower } \\
\text { Bound }\end{array}$ & $\begin{array}{l}\text { Upper } \\
\text { Bound }\end{array}$ & & & $\begin{array}{l}\text { Lower } \\
\text { Bound }\end{array}$ & $\begin{array}{l}\text { Upper } \\
\text { Bound }\end{array}$ \\
\hline 5.5 & .468 & 4.583 & 6.417 & 5 & & & \\
\hline
\end{tabular}

\section{Discussion}

Acute undifferentiated leukemia are diagnosed when the blasts exhibit no lineage-specific antigens [2]. When the blasts express more than one lineage antigens \& is not possible to assign to any particular lineage, it is called MPAL $[2,7]$.

In the majority of patients with acute leukemia, blast cells can be assigned to specific lineage, myeloid, B- or T-lymphoid. However, in approximately $2-5 \%$ of patients, even after comprehensive immunophenotyping by flow cytometry (FCM), lineage of leukemias remains ambiguous. Historically various terminologies were used, such as mixed lineage leukemia, hybrid acute leukemia, bilineal leukemia, and biphenotypic leukemia [2,7]. Diagnostic criteria were modified by WHO classification of hematopoietic and lymphoid tumors (WHO 2008) \&introduced a new designation as mixed-phenotype acute leukemia [2].
As the years passed more antibodies became available $\&$ it was found that aberrant expression of antigens of another lineage was common in acute leukemias. As these leukemias should not be categorized as biphenotypic acute leukemia, many researchers tried to address this issue by allotting a score for these leukemias. Catovsky et al. in 1991, Scoring criteria by European Group for Immunological classification of Acute Leukemia (EGIL), tried to address this issue and to define these leukemias accurately.Various markers were assigned with a score of 2,1 or 0.5 . Score of $>2$ for at least two lineages were classified as biphenotypic acute leukemia. However, this approach was questioned due to the lack of lineage specificity with available markers. Thus in 2008, WHO laid down strict criteria and defined the lineage specific markers for each lineage [7]. 
B cell lineage can be chosen from cCD79a, cCD22, CD24, intracytoplasmic mu chains, CD20 or CD21. The expression of CD10 is also considered as B lineage marker $[2,4]$. So far cCD3 is the strongest marker indicating $\mathrm{T}$ cell lineage [4]. Immunophenotypically Mixed phenotype acute leukemia can be further sub classified as B/Myeloid, T/Myeloid, B/T, or trilineage based on immunophenotypic findings [4]. $\mathrm{B}+\mathrm{T}$ mixed phenotype acute leukemia are extremely rare.

Rarity of B+T MPAL is established by following facts. The largest study so far is by Heidrunn Gerr (2010) et al , he has analysed 92 children, involving multiple centers, over a period of 8 years however no $\mathrm{B}+\mathrm{TMPAL}$ cases were reported [8]. Zhang et al has reported single case of $\mathrm{B}+\mathrm{T}$ MPAL in his series [9]. Present study comprised 10 cases of $\mathrm{B}+\mathrm{T}$ MPAL diagnosis of $\mathrm{B}+\mathrm{TMPAL}$, the largest reported $\mathrm{B}+\mathrm{TMPAL}$ cases till date.

Mixed phenotype acute leukemia is thought to arise from a multi potential hemopoietic stem cell that has the potential to differentiate into any lineage [10]. Most of the reported cases of MPAL, express early hematopoietic markers CD34 positivity. HLADR suggesting an early precursor stem cell origin [10]. In our series, $50 \%$ cases of $\mathrm{T}+\mathrm{B}$ MPAL demonstrated CD34 Positivity

Commonly described cytogenetic groups in MPAL are, compelx karyotypes, $\mathrm{t}(9 ; 22),(\mathrm{q} 34 ; \mathrm{q} 11)$ and $\mathrm{t}(\mathrm{v} ; 11 \mathrm{q} 23)$ [2]. The occurrence of $t(9 ; 22),(q 34 ; q 11)$ and $t(4 ; 11)$ is considered an adverse prognostic factor in ALL patients also Presence of Phildelphia chromosome has been associated with poor prognosis in MPAL [8]. The great majority of, $\mathrm{t}(9 ; 22)(\mathrm{q} 34 ; \mathrm{q} 11)$ positive MPAL are $\mathrm{B}+$ Myeloid [2]. There are occasional case reports of Phildelphia positive B+T MPAL reported [11].

$20 \%$ of B $+\mathrm{T}$ MPAL showed abnormal karyotypes in the present study, however no $\mathrm{Ph}+\mathrm{B}+\mathrm{T}$ MPAL was noted. Single case of Phildelphia positive was noted in B+Myeloid MPAL. Analysis of cytogenetics is becoming increasingly important as potential targets for therapy in future Presence of the phildelphia chromosome should be checked always as it affects the treatment $[2,5]$.

Given the short time these formal criteria have been established and the rarity of MPAL, there is paucity of data regarding the precise biologic characterization of the disease as well as established guidelines on the management and prognosis of these patients [5]. Mixed phenotype acute leukemia is rare leukemias. $\mathrm{B}+\mathrm{T}$ MPAL are uncommon [6], comprises $4 \%$ of MPAL [12]. The lineage specific cytoplasmic markers should be included in the primary panel for categorization of MPAL accurately [7].

There are no set treatment protocols for patients with MPAL, thus evaluation of outcome becomes difficult [13]. The optimal therapeutic approach to B+T MPAL has not been defined. In $41 \%$ patients treated with AML protocol and $85 \%$ patients with ALL treatment, achieved complete remission, In a recently published larger analysis of $100 \mathrm{MPAL}$ patients. The overall median survival was 18 months, in this series. Children had a better survival than adults [7]. Out data suggests that children, who received ALL directed treatment, had a better response to treatment than adults and median survival was 5 years.

Submicroscopic chromosome deletions, amplifications, and unbalanced chromosome rearrangements are recognized frequently by Genomic microarrays in recent years and are widely used to identify cryptic leukemia-related genetic alterations, especially in hematologic malignancies $[13,14]$.

Study by Lingzhiyan et al is the first molecular genomic study of MPAL or biphenotypic leukemia [14]. Out data suggests that $\mathrm{B}+\mathrm{T}$ MPAL are not so rare in Indian population and with ALL directed treatment, have a better response to treatment.

Novel genomic technologies, such as next-generation sequencing, may helpto define the leukemogenic mechanisms in MPAL cases and to determine a standardized treatment for these rare leukemias $[13,14]$.

Molecular explorations will be of great help in understanding of these strange leukemias, however due to financial constraints Gene sequencing was not done in these cases. B+T MPAL are relatively more frequent in India and molecular studies and multi-center studies to determine optimal regimens approach in Indian scenario will be of immense help in understanding these strange leukemias.

Funding: Nil, Conflict of interest: None.

Permission of IRB: Yes

\section{References}


1. Bene MC, Porwit A .Acute leukemias of ambiguous lineage Semin Diagn Pathol, 2012 Feb;29(1): 12-8

2. Borowitz M J Bene MC, HarrisNL, PorwitA, Matutes E Acute leukemia of ambiguous lineage: WHO classification of hematopoietic and lymphoid tissue $4^{\text {th }}$ ed.IARC, Lyon, France:2008.pp 150-155.

3. Ludwig, W.d.,Haferlach,T.\&Schoch,C(2003)Classification of Acute Leukaemias:PerspectiveI. Humana Press Inc., Totowa, NJ.

4. Tseih Sun .Mixed phenotypic acute leukemia: In Flow cytometry, immunohistochemistry and molecular genetics for hematologic neoplasms: $2^{\text {nd }}$ edition. wolters kluwer/Lippincott Williams \& wilkins,Phildelphia.2012; chapter 16:168-173.

5. Sameer kulkarni, Michael Lill and Dimitrios Tzachanis .HSOA Journal of Hematology.Blood transfusion and Disorders.JHematolBloodTransfus Disord2014; vol1:issue1.

6.

EstellaMatutes,RicardoMorilla,NahlaFarahat,FelixCarb onell,JohnSwansbury,Martin Dyer,Daniel Catovsky . Recent Advances in the cyto biology of Leukemias,Definition of Acute BiphenotypicLeukemia.Haematologica 1997; 82(1):6466.

7. Renu Sukumaran, Rekha A Nair, Priya Mary Jacob, Kunjulekshmi Ammaraveen drannair Anila, Shruthy Prem, Rajeshwary Binitha et al .Flowcytometry analysis of Mixed phenotype acute leukemia: Experience from a tertiary oncology center.Indian Journal of Pathology and Microbiology.June 2015;58(2).

8. Heidrun Gerr, Martin Zimmermann, MartinSchrappe, Michael Dworzak, Wolf-Dieter Ludwig, Jutta Bradtke, Anja Moericke, Richard Schabathetal. Acuteleukemias of ambiguous lineage in children: characterization, prognosis and therapy recommendations; British journal of hematology.2010;149(1),84-92. doi 10.1111/j13652141.2009.08058/epub2010jan18

9. Zhang $\mathrm{YM}^{1}, \mathrm{Wu} \mathrm{DP}$, Sun $\mathrm{AN}$ et al. Study on the clinical characteristics of 32 patients with mixed phenotype acute leukemia. ZhonghuaXue Ye XueZaZhi. 2011 Jan;32(1):12-6.

10. Gujral S, Polampalli S, BadrinathY, Kumar A, SubramanianPG,Raje G, et al.Clinico-hematological profile in biphenotypic acute leukemia.Indian Journal of Cancer, April-June, 2009; Vol. 46: No. 2pp. 160-168.

11. Kohla $\mathrm{SA}^{1}$, sabbagh AA ${ }^{1}, \mathrm{Omri} \mathrm{H} \mathrm{E}^{2}$,Ibrahim $\mathrm{F}$ $\mathrm{A}^{1}$,Otazu I B ${ }^{3}$,Alhajri $\mathrm{H}^{3}$ Yassin M A .Mixed Phenotype Acute Leukemia with Two Immunophenotypically Distinct $\mathrm{B}$ and $\mathrm{T}$ Blasts Populations,DoublePh(+) Chromosome and complex Karyotype.Report of an Unusal Case .Clin Med Insights Blood Disord.2015 sept 21 ; 8:25-31. doi:10.4137/CMBD.s24631.c collection 2015.

12. April EstellaMatutes, Winfried FPicki, Marsvan,' Veer, Ricardo Morilla, John Swansbury, Herbert Strobletal Mixed- phenotype acute leukemia: clinical and laboratory features and outcome in 100 patients defined according to the WHO 2008 classification.Blood.2011;volume 117,no11,31633171.doi:10.1182/blood-2010-10-314682.

13. O K Weinberg and DA Arber .Mixed -Phenotype acute leukemia :historical overview and a new definition. Leukemia 2010;24:1844-1851.

14. Lingzhi Yan, Nana Ping, Mingging Zhu, Aining Sun, Yongquan Xue, Changgeng Ruanetal.Clinicalimmunophenotypic, Cytogenetic, And Molecular Genetic Features In 117 Adult Patients With Mixed-Phenotype Acute Leukemia Defined By WHO2008 Classification.Haematologica November 2012 ; 97:

1708-1712.

\section{How to cite this article?}

Raghvendra H Vijay, Namrata N Rajkumar, Vijay C Raghu, Chinnagiriyappa LK. Rare Entities of Mixed Phenotypic Acute Leukemia: Are They Really So Rare? Largest Series of B+T MPAL. Int J Med Res Rev 2016;4(3):401-406. doi: 10.17511/ijmrr.2016.i03.21 